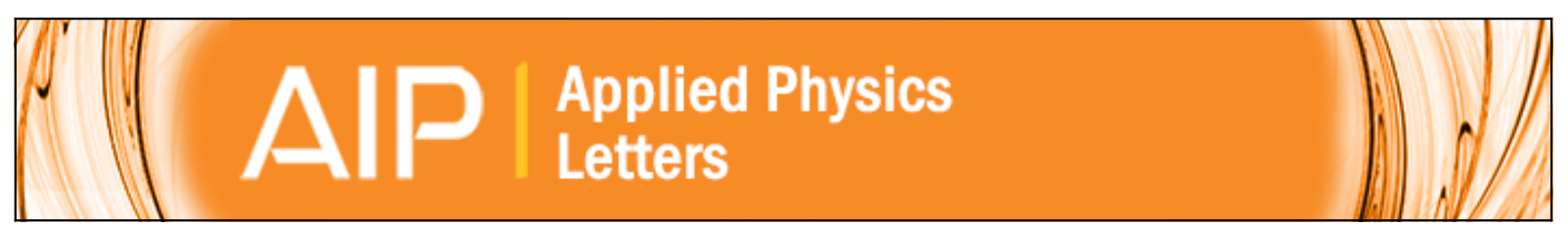

\title{
Local magnetization and strain in single magnetoelectric microrod composites
}

S. B. Hrkac, M. Abes, C. T. Koops, C. Krywka, M. Müller, S. Kaps, R. Adelung, J. McCord, E. Lage, E. Quandt, O. M. Magnussen, and B. M. Murphy

Citation: Applied Physics Letters 103, 123111 (2013); doi: 10.1063/1.4821443

View online: http://dx.doi.org/10.1063/1.4821443

View Table of Contents: http://scitation.aip.org/content/aip/journal/apl/103/12?ver=pdfcov

Published by the AIP Publishing

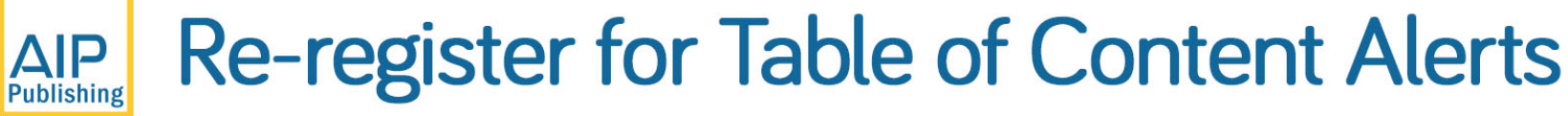

Create a profile.

Sign up today! 


\title{
Local magnetization and strain in single magnetoelectric microrod composites
}

\author{
S. B. Hrkac, ${ }^{1}$ M. Abes,${ }^{1}$ C. T. Koops, ${ }^{1}$ C. Krywka,,${ }^{1,3}$ M. Müller, ${ }^{1,2,3}$ S. Kaps,${ }^{4}$ R. Adelung, ${ }^{4}$ \\ J. McCord, ${ }^{4}$ E. Lage, ${ }^{4}$ E. Quandt, ${ }^{4}$ O. M. Magnussen, ${ }^{1,3}$ and B. M. Murphy ${ }^{1,3, a)}$ \\ ${ }^{1}$ Institute for Experimental and Applied Physics, University of Kiel, 24098 Kiel, Germany \\ ${ }^{2}$ Institute of Materials Research, Helmholtz Zentrum Geesthacht, 21502 Geesthacht, Germany \\ ${ }^{3}$ Ruprecht Haensel Laboratory, University of Kiel, 24098 Kiel, Germany \\ ${ }^{4}$ Institute for Materials Science, University of Kiel, 24143 Kiel, Germany
}

(Received 9 July 2013; accepted 1 September 2013; published online 18 September 2013)

\begin{abstract}
Magneto-optic Kerr effect microscopy and nanofocus X-ray diffraction are combined to investigate the local mapping of the magnetoelectric microcomposite properties of $\mathrm{ZnO}$ microrods coated with an amorphous $\left(\mathrm{Fe}_{90} \mathrm{Co}_{10}\right)_{78} \mathrm{Si}_{12} \mathrm{~B}_{10}$ layer. We follow the magnetic domain behavior and lattice deformation upon applying an external magnetic field. In addition to the expected field induced strain, we observe a local magnetic induced strain in the $10^{-5}$ range in the $\mathrm{ZnO}$ localized near the $\left(\mathrm{Fe}_{90} \mathrm{Co}_{10}\right)_{78} \mathrm{Si}_{12} \mathrm{~B}_{10} / \mathrm{ZnO}$ interface. (C) 2013 AIP Publishing LLC. [http://dx.doi.org/10.1063/1.4821443]
\end{abstract}

Local information on field induced strain and magnetic behavior in magnetoelectric (ME) multiferroic composites is essential to fully understand and thus develop their applications as multifunctional devices. ${ }^{1-3}$ These exciting systems have high potential for biomedical sensor applications ${ }^{4,5}$ such as magnetoencephalography and magnetocardiography. ${ }^{6}$ In ME composites, consisting of a piezoelectric (PE) and a magnetostrictive (MS) material, the magnetic field induced strain is transferred from the MS component to the PE component resulting in electrical polarization and thus a voltage or charge signal. ${ }^{2,3}$ The ME effect is the product of the MS properties, the PE properties, the geometry, the elastic properties, and additionally, the mechanical coupling at the interface. Particular promising are composites of simple piezoelectrics, e.g., $\mathrm{ZnO}$ or $\mathrm{AlN}$, with $\mathrm{MS}$ alloys, such as $\left(\mathrm{Fe}_{90} \mathrm{Co}_{10}\right)_{78} \mathrm{Si}_{12} \mathrm{~B}_{10}$ (Metglas) ${ }^{7}$ or $\mathrm{FeTb}^{8}{ }^{8}$ which show excellent ME effects for low magnetic field applications due to their high permeability and low saturation field.

In the vast majority of studies, macroscopic $2-2$ composites with a layered structure have been employed, due to their easy fabrication and conceptual simplicity. However, micro- or nanostructured ME composites with alternative geometries (e.g., 1-3 composites) gain increasing attention, because of potential substantial increase in sensor sensitivity. For example, studies of a single macroscopic $\mathrm{Pb}(\mathrm{Zr}, \mathrm{Ti}) \mathrm{O}_{3}$ rod embedded in a Terfenol-D/epoxy mixture reported an increase in the ME coefficient by almost a factor of two upon doubling the aspect ratio from 5 to 10 (Ref. 9), and in total a factor of three for an aspect ratio of 24 (Ref. 10). This indicates that major enhancement of ME effects is possible in such isolated 1-3 structures, motivating the studies of high aspect ratio MS Metglas/PE $\mathrm{ZnO}$ microcomposites presented here.

While in 2-2 systems, the field induced strain only varies along the direction normal to the interface; quasi-1D rod like ME composites exhibit a more complex response, involving a 3D distribution of magnetization and strain. Optimization of these structures for sensor applications requires detailed

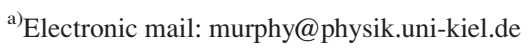

understanding of this behavior, specifically spatially resolved structural and magnetic data. Those cannot be obtained by conventional approaches, where typically indirect measurements of macroscopic changes in magnetization and sample shape are performed (e.g., vibrating sample magnetometer and cantilever bending). ${ }^{11}$ Therefore, in this work, we combine magneto-optic Kerr effect (MOKE) microscopy and nanofocus X-ray diffraction for the local mapping of the ME microcomposite properties. As shown in recent grazing incidence X-ray diffraction (GID) experiments of simple planar 2-2 Metglas/ZnO composites, X-ray diffraction methods allow determination of the magnetic field induced strain in a ME microcomposite directly. ${ }^{12,13}$ Employing the brilliant submicron X-ray beams provided by third-generation synchrotron sources high-resolution strain mapping is possible, as used exemplary here for the study of ME composites based on $\mathrm{ZnO}$ microrods.

The investigated PE microrods, synthesized by flame transport synthesis, ${ }^{14,15}$ have a characteristic hexagonal cross section, are approximately $700 \mu \mathrm{m}$ long, and have a maximum diameter of $27 \mu \mathrm{m}$, corresponding to an aspect ratio of $\sim 26$, i.e., even higher than the aspect ratio given in Ref. 10 (Fig. 1(a)). The prism surfaces correspond to the (100), (010), and (1-10) orientations of the $\mathrm{ZnO}$ crystal. ${ }^{16} \mathrm{ME}$ microcomposites were prepared by coating these microrods with an amorphous Metglas layer of about $0.2 \mu \mathrm{m}$ thickness (an example of a coated $\mathrm{ZnO}$ rod is shown in the right scanning electron microscope (SEM) image in Fig. 1(a)). The layer was deposited by magnetron sputtering while turning the sample to ensure all sides were coated. An in-plane magnetic field of $10 \mathrm{mT}$ was applied during film deposition ${ }^{7}$ perpendicular to the orientation of the rod, in order to induce a magnetic anisotropy perpendicular to the rod axis.

Longitudinal MOKE microscopy ${ }^{17}$ images (Fig. 1(b)) made on an Evico magnetics Kerr microscope ${ }^{18}$ reveal a distinct magnetic domain structure within the MS Metglas film. In the absence of an external magnetic field-i.e., in the demagnetized state (Fig. 1(b) left) - micron sized magnetic domains with antiparallel alignment of the in-plane magnetization are visible, separated by $180^{\circ}$ magnetic domain walls. 
(a)

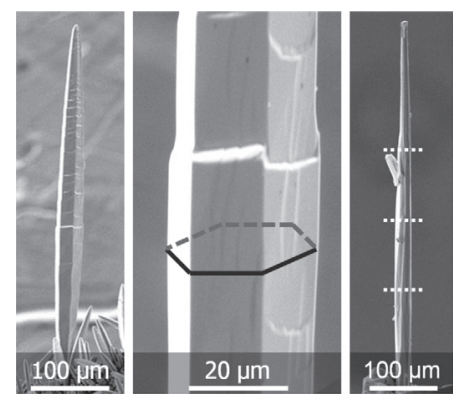

(c)

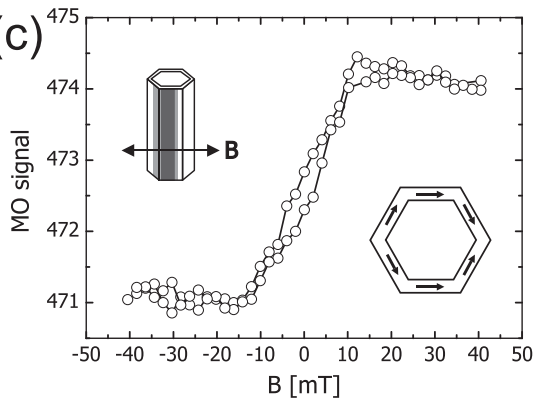

(b)

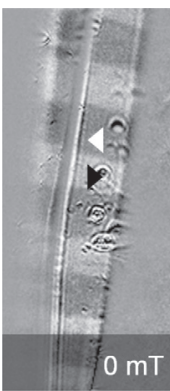

(d)

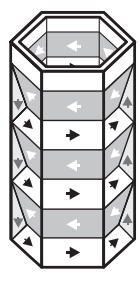

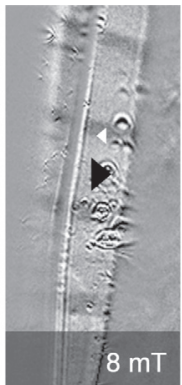
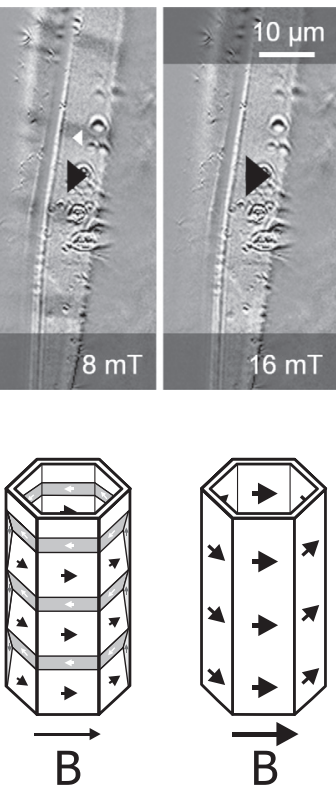

$0 \mu \mathrm{m}$

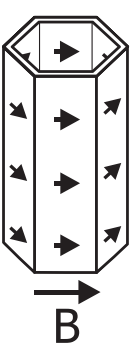

FIG. 1. (a) SEM images of uncoated (left and middle) and Metglas coated (right) $\mathrm{ZnO}$ microrods. The dotted lines show scan positions for the X-ray measurements. (b) MOKE microscope images of the magnetic domains and (c) corresponding magnetization loop in the Metglas coating on a $\mathrm{ZnO}$ microrod. Insets in (c) show schematically the direction of the external magnetic field across the sample and the domain structure at saturation magnetization. (d) Schematic model of the magnetic domain structure, consisting of antiparallel aligned in-plane domains which are separated by $180^{\circ}$ domain walls, in the demagnetized sample, an intermediate state at low magnetic fields, and at saturation.
This behavior is expected as the magnetic field bias was applied perpendicular to the microrod axis during deposition. ${ }^{19}$ Additional stress induced anisotropy contributions cannot be ruled out. Judged from the approximately equal areas of the two domain types, the Metglas coating is largely demagnetized. With the application of a transverse magnetic field $(\mathrm{B}=10 \mathrm{mT})$, the remagnetization process takes place via domain wall motion in the optically visible microrod surface. This process continues with increasing magnetic field, resulting in a single domain state in the MOKE images at $\mathrm{B} \geq 15 \mathrm{mT}$ that suggests the magnetic saturation of the sample along the magnetic field. The magneto-optic hysteresis loop of the microrod coating, obtained from these images, confirms the continuous change in magnetization and the saturation field strength of $15 \mathrm{mT}$ (Fig. 1(c)). The continuous change of magnetization by $180^{\circ}$ domain wall motion along the magnetic easy axis of the system indicates a more complex magnetization process as visible at the top surface. The magnetic reversal behavior along the effective easy axis of magnetization is similar to a patterned ferromagnetic sample due to the demagnetization effects. ${ }^{20}$ From that, these data allow derivation of the field dependent magnetic domain structure for the whole microrod surface, despite the fact that only one surface of the microrod is accessible by the MOKE observations. Specifically, for the given geometry, the continuous change and low hysteresis can only be rationalized by a domain structure involving terminating $90^{\circ}$ domains or similar closure domain states with magnetization in [001] direction at the outer corners of the hexagonal microrods, which are gradually removed with increasing magnetic field (schematically shown in Fig. 1(d)). At saturation, a head-tohead and tail-to-tail magnetization states occur.

The associated local magnetization rotation during changes in the external magnetic field induces strain in the MS layer, leading to lattice deformation within the $\mathrm{ZnO}$ microrod. The latter is probed via X-ray diffraction at an energy of $12.8 \mathrm{keV}$ provided by the high resolution nanofocus endstation of the MiNaXS-Beamline P03 at PETRA $\mathrm{III}^{21}$ where a focusing mirror optics provided an X-ray beam of $400 \mathrm{~nm} \times 800 \mathrm{~nm}$ size (horizontal and vertical, respectively). The samples were mounted on a Huber goniometer head which in turn was mounted on a motor to enable sample rotation around the $c$-axis providing access to $\mathrm{ZnO}(h 0 l)$ Bragg reflections with $h$ and $l=-1,0$, and 1 . With the sample in transmission geometry (Fig. 2(a)) and employing a 2D Dectris $300 \mathrm{k}$ Pilatus Pixel detector (pixel size of $172 \times 172 \mu \mathrm{m}^{2}$ ) at a distance of $185 \mathrm{~mm}$ from the sample, we measure the diffraction angle of the $\mathrm{ZnO}(100)$ Bragg reflection in the presence of an external magnetic field (corresponding macroscopic and atomic scale diffraction geometries are shown schematically in Figs. 2(b) and 2(c), respectively). The diffraction patterns were analysed using the European Synchrotron Radiation Facility software FIT2D. ${ }^{22}$

The (100) Bragg reflection is always intense when the nanobeam hits the microrod, confirming the homogeneity of the crystal. A characteristic example of the measured diffracted intensity at $\mathrm{ZnO}(100)$ as a function of the scattering vector in $(h 00)$ direction $q_{\perp}$ is presented in Fig. 2(d). The full width half maximum of the Bragg peak at the reciprocal space position $q_{100}$ is $\Delta q_{\perp} \sim 0.0077 \pm 0.0001 \AA^{-1}$, illustrating the very high crystal quality of the $\mathrm{ZnO}$ rods and indicating a crystal domain size of $1500 \pm 70 \AA$, neglecting other defects and strain using the Scherrer equation. ${ }^{23}$ By monitoring $q_{100}$ as a function of the nanobeam position perpendicular ( $y$ in $1 \mu \mathrm{m}$ steps) and parallel ( $z$ in $100 \mu \mathrm{m}$ steps) to the microrod axis, the local $\mathrm{ZnO}$ lattice spacing $d_{100}$ can be mapped with high spatial resolution. Using these steps and the beam size, there is no overlaying effect between spots. The in-plane geometry as seen in Fig. 2(a) shows the incident beam $\boldsymbol{k}_{\mathbf{0}}$ impinging in the sample under the Bragg angle of $\theta=10.092^{\circ}$ relative to the (100) planes.

For the Metglas coated $\mathrm{ZnO}$ rod shown in Fig. 1(a) (right), we show characteristic local dependencies $d_{100}(y)$ at three different $z$ positions along the rod (indicated in Fig. 1(a) by white dashed lines), offset with distances $\Delta z=100 \mu \mathrm{m}$, in Fig. 3. Clear variations of $d_{100}$ are visible in contrast to bare $\mathrm{ZnO}$ microrods, which show a highly uniform lattice spacing 
(a)

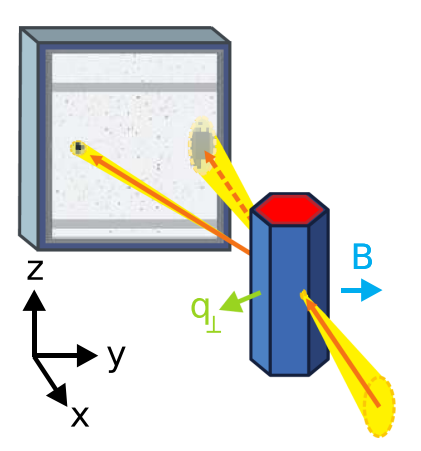

(b)

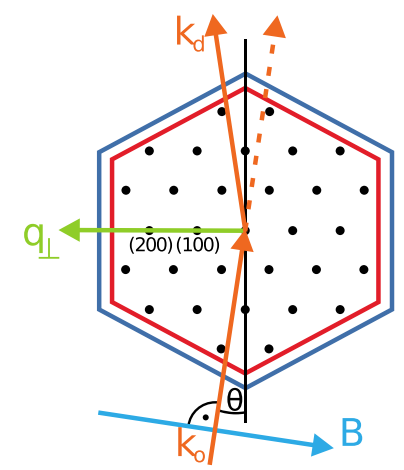

(c)

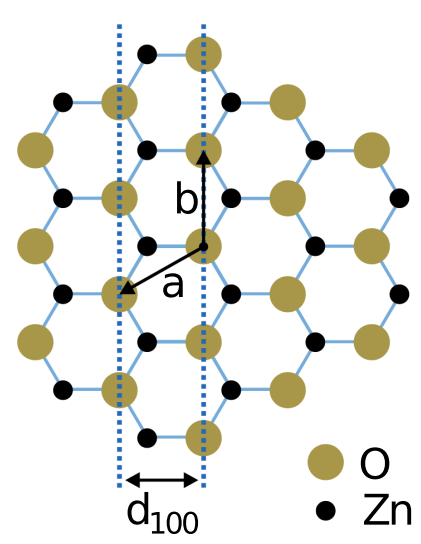

(d)

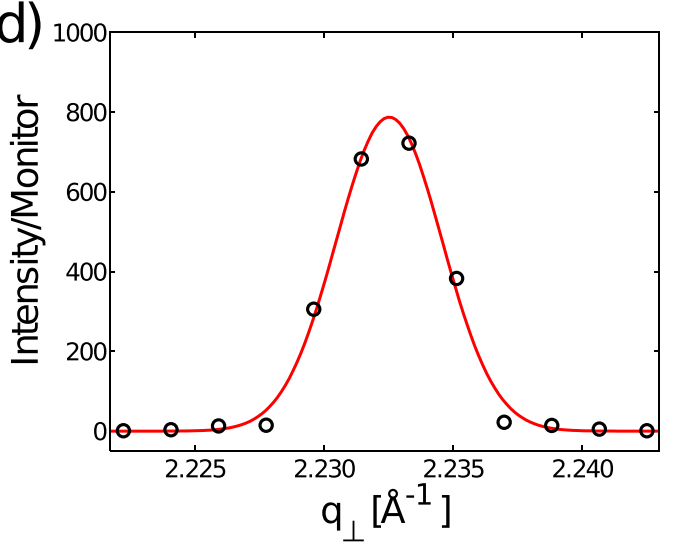

FIG. 2. (a) and (b) Schematic view of the in-situ nanofocus $X$-ray diffraction geometry. (c) $\mathrm{ZnO}$ lattice structure in the basal plane. (d) Example of the $\mathrm{ZnO}$ (100) Bragg reflection measured on the $\mathrm{ZnO}$ rod by nanofocus diffraction (errors are smaller than the symbol size). $\left(d_{100}=2.8159 \pm 0.0001 \AA\right)$ across the whole rod, illustrating the absence of intrinsic strain (Fig. S1, Ref. 24). The growth induced static strain $\varepsilon_{(\mathrm{c}) 100}$, along the [h00] direction, can be calculated from the uncoated $\left(q_{100}(\mathrm{uc})\right)$ and the Metglas coated $\left(q_{100}(c)\right) \mathrm{ZnO}$ Bragg reflection positions via

$$
\varepsilon_{(c) 100}=\left(q_{100}(u c) / q_{100}(c)\right)-1 .
$$

The entire Metglas coated samples exhibit a large compressive strain in average $\varepsilon_{(\mathrm{c}) 100}=-55 \times 10^{-5}$. The intrinsic strain varies by $\Delta \varepsilon_{(\mathrm{c}) 100}= \pm 20 \times 10^{-5}$ (Fig. S2, Ref. 24) near the edges of the sample, i.e., under conditions when the beam predominantly probes $\mathrm{ZnO}$ areas near the (100) prism faces (Fig. 2(b); the systematic differences between left and right sample side are due to the diffraction geometry). This compressive strain is caused by the mechanical stress of the coating material on the $\mathrm{ZnO}$ crystal during the sample preparation. A similar effect was recently observed in planar $\mathrm{FeTb} / \mathrm{ZnO} \mathrm{ME}$ composites ${ }^{13}$ prepared by an analogue method. However, while in those 2-2 composites, the strain extended up to $15 \mu \mathrm{m}$ into the $\mathrm{ZnO}$ bulk, the corresponding strain decay in the microcomposite occurs over two length scales, initially, a sharp decay within about the first $5 \mu \mathrm{m}$ (Fig. 3(a)) and a residual strain remaining over the entire rod. This indicates a stronger localization of the strain near the ME interface in the 1-3 type of composites, illustrating the substantial influence of the microstructure geometry on the strain distribution. Along with the strain variation across the rod, different $z$ positions also exhibit significant local strain differences. Variation in the domain structure along the z-direction can be ruled out as the cause, since these variations remain visible even at high magnetic fields. These variations are instead attributed to inhomogeneities in the Metglas coating and demonstrate the need for highly spatially resolved strain mapping in the characterization of these microcomposites. Studies of magnetic field induced strain were performed at the same locations on the sample with external magnetic fields applied perpendicular to the incident beam and the microrod axis (Figs. 2(a) and 2(b)) using motorized permanent magnets calibrated with a teslameter. As shown in Fig. 3(a), the strain distribution of the ME composite is clearly modified in a field of $10 \mathrm{mT}$. In contrast, for the pure (uncoated) $\mathrm{ZnO}$ rods, the Bragg reflections positions remain independent of the applied magnetic field clearly showing that the effect is a direct result of the MS response of the Metglas mantle. The resulting local distributions of magnetic field induced $\Delta \varepsilon_{(\mathrm{B}) 100}=\varepsilon_{(\mathrm{B}) 100}-\varepsilon_{(\mathrm{B}=0) 100}$ along the three line scans across the $\mathrm{ZnO}$ rod are displayed in Fig. 3(b). Whereas the strain in the center of the rod remains almost unchanged upon applying the field, with an average value of $\Delta \varepsilon_{(\mathrm{B}) 100}=(1 \pm 1) \times 10^{-5}$, substantial changes are found near the sample edges. Overall, $\Delta \varepsilon_{(\mathrm{B}) 100}$ decays from a local maximum of up to $\Delta \varepsilon_{(\mathrm{B}) 100}=(10 \pm 2) \times$ $10^{-5}$ near the edges toward the sample center. This decay occurs on similar length scales as the initial decay in the static strain distributions at $B=0 \mathrm{mT}$. Furthermore, the (tensile) field induced strain at a given sample position as compared to the preexisting static (compressive) strain is typically 60 times smaller at the center of the rod and 6 to 8 times smaller in the interface region. All these observations strongly indicate that the main magnetic field induced effect is a relaxation of the deposition-induced strain with an enhancement at the interface. In good agreement with the MOKE measurements seen in Fig. 1(c), measurements at variable field strengths show that the changes in $\Delta \varepsilon_{(\mathrm{B}) 100}$ are reversible and reach saturation within the experimental error $^{25}$ at about $10 \mathrm{mT}$ (Fig. 3(c)) (Fig. S3, Ref. 24). As in the case of static strain, $\Delta \varepsilon_{(\mathrm{B}) 100}$ exhibits substantial spatial 


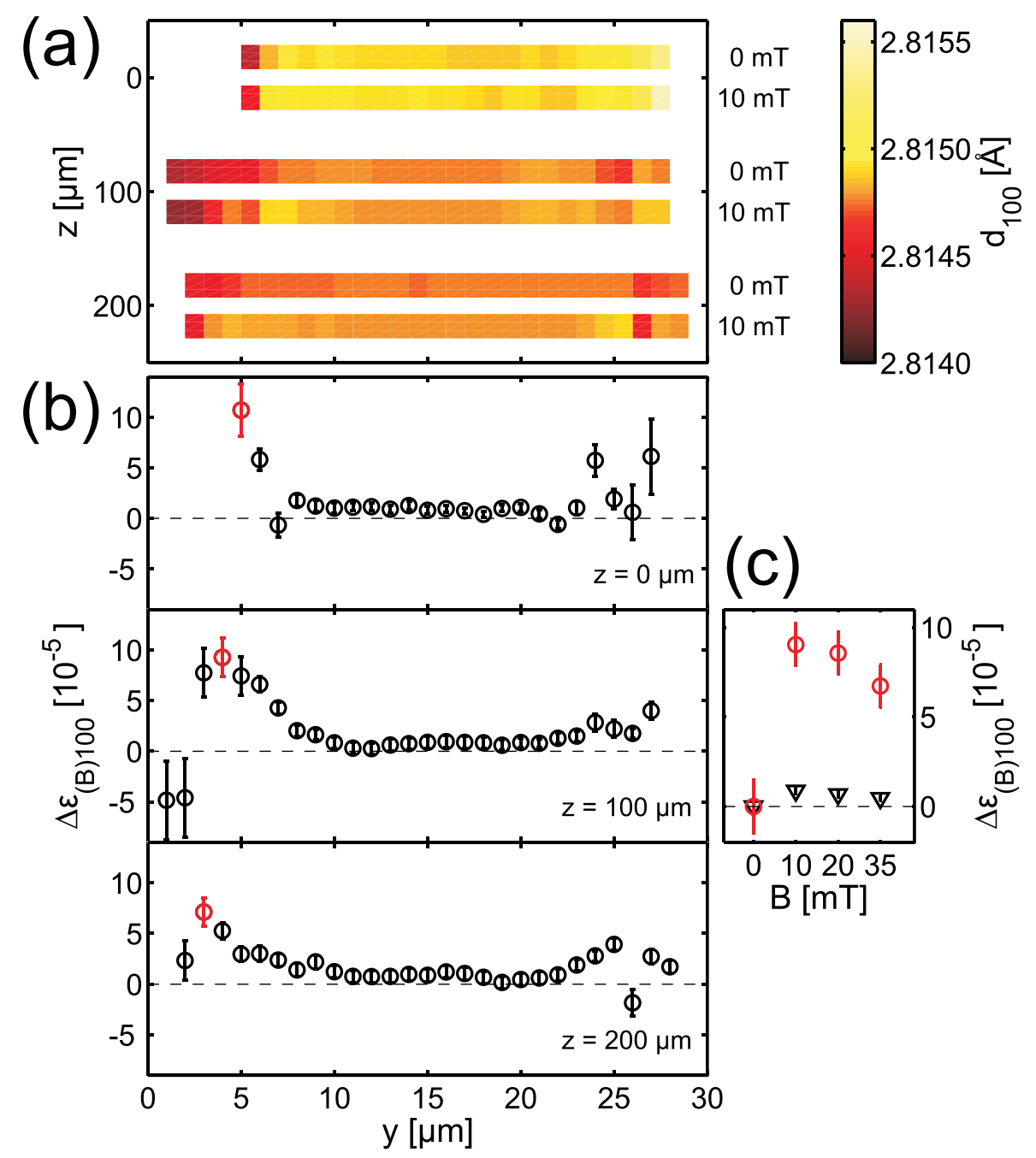

FIG. 3. (a) Map of the local lattice spacing $d_{100}$, obtained from the positions of the (100) Bragg reflection, and (b) corresponding local profiles of the magnetic field induced strain $\Delta \varepsilon_{100}$ in the Metglas-coated $\mathrm{ZnO}$ microrod at these locations in $y$ and $z$ at $10 \mathrm{mT}$. (c) Average field induced strain $\Delta \varepsilon_{(\mathrm{B}) 100}$ at the local maxima at the edge (red symbol in (b)) and the average value of the strain between $y=10 \mu \mathrm{m}$ and $y=20 \mu \mathrm{m}$ across all three lines (black inverted triangle) as a function of applied magnetic field.

fluctuations, including areas with local enhancement of the compressive strain (i.e., with $\Delta \varepsilon_{(\mathrm{B}) 100}<0$ ), which again is attributed to sample inhomogeneities.

The high local maximum of the field induced strain

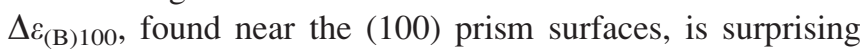
and not easily rationalized by conventional considerations. The MOKE observations reveal an easy axis of magnetization in the Metglas film that is perpendicular to both the microrod axis and to the [100] direction of the $\mathrm{ZnO}$ substrate, i.e., the [120] direction. Considering geometry and perfect strain coupling, the maximum strain should not exceed $3 / 2 \lambda_{S}{ }^{20}$ which is $4 \times 10^{-5},{ }^{12}$ where $\lambda_{S}$ is the saturation magnetostriction constant of bulk amorphous Metglas. ${ }^{20}$ According to the nanodiffraction measurements, the local field induced strain in the microcomposite can exceed this value by more than a factor of two. In contrast, our previous GID studies of analogue 2-2 composites, ${ }^{12}$ consisting of $200 \mathrm{~nm}$ Metglas films on macroscopic $\mathrm{ZnO}$ single crystals do not indicate a comparable strain enhancement. In those planar samples, which were prepared in an identical way and therefore can be directly compared with the microcomposites, the field induced strain in the $\mathrm{ZnO}$ near the interface saturates at about $3 \times 10^{-5}$.

Several factors could account for the significant enhancement of the field induced strain in the microrod composites. Firstly, it might be related directly to the specific geometry of these systems. For example, it could be caused by an overlap of the strain fields, emerging from the (100) and the adjacent prism faces. Second, the very substantial (deposition induced) strain in the sample at zero field has to be considered. The strain change $\Delta \varepsilon_{(\mathrm{B}) 100}$ even at saturation field is much smaller than this static strain. It may be considered as a field induced relaxation of the latter, which could exceed the magnetostriction of an initially strain-free sample. To clarify to which extent these effects contribute to the strain enhancement will require more systematic studies of ME microrods as a function of the structure size (e.g., diameter, coating thickness) and initial preparation induced strain.

In summary, the Metglas/ZnO microcomposite structures studied here display unusual, interesting properties for ME applications. In particular, the nanofocus diffraction studies show that the MS strain changes induced in the PE component can indeed be enhanced in such structures, as suggested by previous more macroscopic ME measurements. ${ }^{2,26,27}$ The initial strain and also the field induced changes in the $\mathrm{ZnO}$ are localized near the Metglas/ZnO interface, with the latter being well correlated with the magnetization behavior observed by MOKE microscopy. The possibility to determine in single ME microcomposites both the magnetization and strain changes in the $10^{-5}$ range with micron spatial resolution, as demonstrated in this work, is crucial for understanding these 
complex systems and will also be highly useful for studies of other similar functional microsystems.

The authors thank the German Science Foundation (DFG) for financial support via the Collaborative Research Centre SFB 855 "Magnetoelectric Composite Materials Biomagnetic Interfaces of the Future" and the BMBF via grants $05 \mathrm{~K} 10 \mathrm{FK} 3$ and $05 \mathrm{KS} 7 \mathrm{FK} 3$, and DESY for funding the nanofocus setup at P03. We also thank the staff of the P03 beamline at PETRA III; Matthias Greve at University Kiel; and Judith Bach, Robert Frömter, and Hans Peter Oepen at University Hamburg for technical assistance.

${ }^{1}$ M. H. Phan and H. X. Peng, Prog. Mater. Sci. 53, 323 (2008).

${ }^{2}$ C. W. Nan, M. I. Bichurin, S. X. Dong, D. Viehland, and G. Srinivasan, J. Appl. Phys. 103, 031101 (2008).

${ }^{3}$ J. Ma, J. M. Hu, Z. Li, and C. W. Nan, Adv. Mater. 23, 1062 (2011).

${ }^{4}$ R. Jahns, H. Greve, E. Woltermann, E. Quandt, and R. H. Knochel, IEEE Trans. Instrum. Meas. 60, 2995 (2011).

${ }^{5}$ J. Y. Zhai, Z. P. Xing, S. X. Dong, J. F. Li, and D. Viehland, Appl. Phys. Lett. 88, 062510 (2006).

${ }^{6}$ M. Hamalainen, R. Hari, R. J. Ilmoniemi, J. Knuutila, and O. V. Lounasmaa, Rev. Mod. Phys. 65, 413 (1993).

${ }^{7}$ H. Greve, E. Woltermann, H. J. Quenzer, B. Wagner, and E. Quandt, Appl. Phys. Lett. 96, 182501 (2010).

${ }^{8}$ E. Quandt, A. Ludwig, D. G. Lord, and C. A. Faunce, J. Appl. Phys. 83, 7267 (1998).

${ }^{9}$ J. Ma, Z. Shi, and C. W. Nan, Adv. Mater. 19, 2571 (2007).

${ }^{10}$ J. Ma, Z. Shi, and C. W. Nan, J. Phys. D: Appl. Phys. 41, 155001 (2008).

${ }^{11}$ E. du Trémolet de Delacheisserie and J. C. Peuzin, J. Magn. Magn. Mater. 136, 189 (1994).

${ }^{12}$ M. Abes, C. T. Koops, S. Hrkac, E. Woltermann, H. Greve, E. Quandt, S. P. Collins, O. M. Magnussen, and B. M. Murphy, Appl. Phys. Lett. 102, 011601 (2013).
${ }^{13}$ M. Abes, C. T. Koops, S. B. Hrkac, E. Quandt, L. Bouchenoire, B. M. Murphy, and O. M. Magnussen, J. Appl. Phys. 113, 124303 (2013).

${ }^{14}$ S. Kaps, R. Adelung, C. Wolpert, T. Preuße, and Y. K. Mishra, "Elastisches Material mit einem auf Partikelebene durch Nanobrücken zwischen Partikeln überbrückten Porenraum," Deutsches Patentamt DE2011000282 (September 29, 2011).

${ }^{15}$ Y. K. Mishra, R. Adelung, C. Rohl, D. Shukla, F. Spors, and V. Tiwari, Antiviral Res. 92, 305 (2011).

${ }^{16}$ Z. L. Wang, Mater. Sci. Eng. R. 64, 33 (2009).

${ }^{17}$ A. Hubert and R. Schäfer, Magnetic Domains: The Analysis of Magnetic Microstructures (Springer-Verlag, Berlin, 1998), p. 21.

${ }^{18}$ B. E. Argyle and J. McCord, Efficient Kerr Microscopy, Magnetic Storage Systems Beyond 2000, NATO Science Series (Springer, 2001), Vol. 41, p. 287.

${ }^{19}$ M. Ali, R. Watts, W. J. Karl, and M. R. J. Gibbs, J. Magn. Magn. Mater. 190, 199 (1998)

${ }^{20} \mathrm{E}$. Du Trémolet De Lacheisserie, Magnétisme, Matériaux et Applications (EDP Sciences, Grenoble, 1999), p. 362.

${ }^{21}$ C. Krywka, H. Neubauer, M. Priebe, T. Salditt, J. Keckes, A. Buffet, S. V. Roth, R. Doehrmann, and M. Mueller, J. Appl. Crystallogr. 45, 85 (2012).

${ }^{22}$ A. P. Hammersley, S. O. Svensson, M. Hanfland, A. N. Fitch, and D. Hausermann, High Press. Res. 14, 235 (1996).

${ }^{23}$ B. E. Warren, X-Ray Diffraction (Addison-Wesley, Reading, Massachusetts, 1969), p. 253.

${ }^{24}$ See supplementary material at http://dx.doi.org/10.1063/1.4821443 for indepth information on internal and magnetic field induced strain of pure and coated $\mathrm{ZnO}$ rods at different magnetic fields.

${ }^{25}$ The strain changes $\Delta \varepsilon_{(\mathrm{B}) 100}$ near the sample edge (red circles) seem to slightly decrease with increasing magnetic field for $\mathrm{B} \geq 20 \mathrm{mT}$. At present, it is not clear whether this effect is real due to the large experimental errors. It might be caused by the pronounced local strain variations and instrumental effects, e.g., field-induced mechanical motion of the sample.

${ }^{26}$ Z. Fang, S. G. Lu, F. Li, S. Datta, Q. M. Zhang, and M. El Tahchi, Appl. Phys. Lett. 95, 112903 (2009).

${ }^{27}$ M. I. Bichurin, V. M. Petrov, and G. Srinivasan, Phys. Rev. B 68, 054402 (2003). 